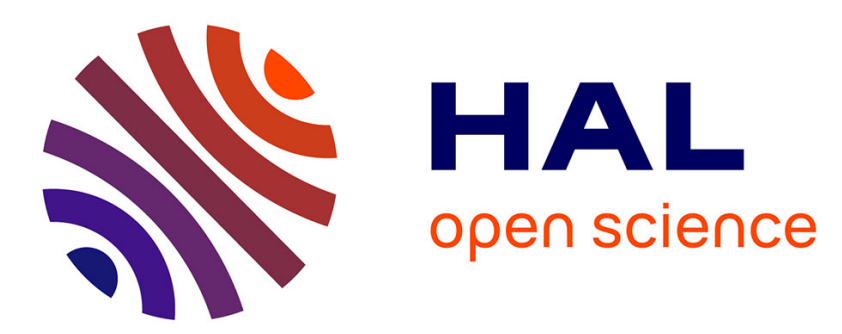

\title{
The relevance of the artery of Adamkiewicz for microsurgical resection of spinal tumors- short overview and case series: Technical note
}

Constantin Tuleasca, Anne Herbrecht, Steven Knafo, Nozar Aghakhani, Fabrice Parker

\section{To cite this version:}

Constantin Tuleasca, Anne Herbrecht, Steven Knafo, Nozar Aghakhani, Fabrice Parker. The relevance of the artery of Adamkiewicz for microsurgical resection of spinal tumors- short overview and case series: Technical note. Neurochirurgie, In press, 10.1016/j.neuchi.2020.07.001 • hal-02924769

\section{HAL Id: hal-02924769 \\ https://hal.sorbonne-universite.fr/hal-02924769}

Submitted on 28 Aug 2020

HAL is a multi-disciplinary open access archive for the deposit and dissemination of scientific research documents, whether they are published or not. The documents may come from teaching and research institutions in France or abroad, or from public or private research centers.
L'archive ouverte pluridisciplinaire HAL, est destinée au dépôt et à la diffusion de documents scientifiques de niveau recherche, publiés ou non, émanant des établissements d'enseignement et de recherche français ou étrangers, des laboratoires publics ou privés. 


\section{Journal Pre-proof}

The relevance of the artery of Adamkiewicz for microsurgical resection of spinal tumors- short overview and case series: technical note

Constantin Tuleasca MD PhD Anne Herbrecht MD Steven Knafo MD PhD Nozar Aghakhani MD PhD Fabrice Parker MD PhD

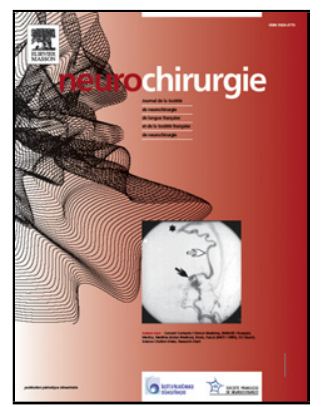

PII:

S0028-3770(20)30360-X

DOI:

https://doi.org/doi:10.1016/j.neuchi.2020.07.001

Reference:

NEUCHI 1118

To appear in:

Neurochirurgie

Received Date:

3 June 2020

Revised Date:

22 June 2020

Accepted Date:

27 July 2020

Please cite this article as: Tuleasca C, Herbrecht A, Knafo S, Aghakhani N, Parker F, The relevance of the artery of Adamkiewicz for microsurgical resection of spinal tumors- short overview and case series: technical note, Neurochirurgie (2020), doi: https://doi.org/10.1016/j.neuchi.2020.07.001

This is a PDF file of an article that has undergone enhancements after acceptance, such as the addition of a cover page and metadata, and formatting for readability, but it is not yet the definitive version of record. This version will undergo additional copyediting, typesetting and review before it is published in its final form, but we are providing this version to give early visibility of the article. Please note that, during the production process, errors may be discovered which could affect the content, and all legal disclaimers that apply to the journal pertain.

(C) 2020 Published by Elsevier. 


\section{The relevance of the artery of Adamkiewicz for microsurgical resection of spinal} tumors- short overview and case series: technical note

Importance de l'artère de l' Adamkiewicz pour la resection microchirurgicale des tumeurs intramédullaires- à propos d'une série de cas

Constantin Tuleasca*1,2,3,4,5 , MD-PhD, Anne Herbrecht*5, MD, Steven Knafo ${ }^{5}$, MD, PhD, Nozar Aghakhani ${ }^{5, *}, \mathrm{MD}, \mathrm{PhD}$ and Fabrice Parker ${ }^{5, *}, \mathrm{MD}, \mathrm{PhD}$

${ }^{1}$ Department of Clinical Neurosciences, Neurosurgery Service and Gamma Knife Center, Lausanne University Hospital (CHUV), ${ }^{2}$ University of Lausanne (Unil), Faculty of Biology and Medicine (FBM), ${ }^{3}$ Signal Processing Laboratory (LTS 5), Ecole Polytechnique Fédérale de Lausanne (EPFL), ${ }^{4}$ Sorbonne Université, Faculté de Médecine, ${ }^{5}$ Assistance Publique-Hôpitaux de Paris, Hôpitaux Universitaires Paris-Sud, Centre Hospitalier Universitaire Bicêtre, Service de Neurochirurgie, Paris, France;

* Dr Tuleasca and Herbrecht equally contributed to the present manuscript as first authors

\section{Corresponding author:}

Constantin Tuleasca, MD-PHD, Centre Hospitalier Universitaire de Lille, Hôpital Roger Salengro; Centre Hospitalier Universitaire Vaudois, Neurosurgery Service and Gamma Knife Center, Rue du Bugnon 44-46, BH-08, CH-1011, Lausanne, Switzerland Tel: +41-21-314-26-02; Fax: +41-21-314-11-99; e-mail: constantin.tuleasca@chuv.ch, constantin.tuleasca@gmail.com

\section{Abstract}

Introduction: There is of major interest to know the exact anatomical location of artery of Adamkiewicz (AKA) for many spinal microsurgical procedures, so as to avoid postoperative ischemia of the spinal cord, with further devastating clinical impact.

Methods and results: We detail the interest of preoperative angiography for medullary lesions in our experience. We further report two cases where the AKA was located at the 
same level and side with the treated lesion. The first case underwent an intracapsular decompression. The second patient undertook radiosurgery by Cyberknife.

Conclusion: For spinal tumor where the AKA is exactly on the same level and side, an intracapsular decompression can be safely performed, with an immediate decrease of the symptomatic mass effect, while decreasing the risk of neurological injury. A second valuable alternative in such situations can also be radiosurgery, for small to medium size tumors. In our experience, this proved safe and effective both for tumor and eventual pain control.

Key words: Adamkiewicz, spinal tumors, neurinoma, radiosurgery, schwannoma, Cyberknife Short title: Artery of Adamkiewicz for microsurgical resection of spinal tumors

\section{Introduction}

Spinal cord vascularization relies on 3 main arteries: the anterior spinal (ASA, supplying the anterior two thirds) and the two postero-lateral spinal (supplying the posterior third)(1). The ASA originates at the level of the foramen magnum, from the 2 vertebral arteries. Anterior segmental medullary vessels from the aorta further supply it, the biggest of which is the artery of Adamkiewicz (AKA, from the descending aorta)(1). The AKA classically rises from the left side of the aorta, between T8 and L2, frequently T9-T12, in a vast majority of $80 \%$ of cases. However, it might be found above T8 in approximately $15 \%$ of people. Anatomical variations include: arising from the right side, levels outside T8 to L2, variances in the angle of how the AKA joins the anterior spinal artery, or presence of more than one AKA. As the AKA is the main arterial supply for the entirety of the lower thoracic cord, the former has been traditionally been seen as a watershed area.

There is of major interest to know the exact location of AKA for many surgical spinal procedures, to avoid postoperative ischemia of the spinal cord, with their further clinical impact (see below). Our department is specialized, among other procedures, on the resection of spinal tumors, including intramedullary lesions. In this context, the preoperative anatomical evaluation of AKA via computer tomography (CT-angio)(2), magnetic resonance imaging 
(MRI) or spinal angiography (SA, depending on the case) is, for us, of crucial importance(3). Some indications for spinal angiography, in our experience, can be seen in figure 1. Are illustrated vascular lesions (Figure 1, A), endocanalar juxtaforaminal lesions (Figure 1, B), foraminal lesions (Figure 1, C) or dumbell lesions (Figure 1, D).

\section{Surgical considerations}

The precise position of AKA is extremely important for many surgical procedures of several subspecialties, not limited to neurosurgery only. Lack of flow within this vessel can cause ischemia to the spinal cord (see below). Care should be given in neurosurgery (resection of intramedullary tumors, thoracolumbar spine surgery) but also in vascular surgery (thoracoabdominal aortic aneurysm repair), urology and pediatric surgery (retroperitoneal dissections)(4).

In a recent study, the dural crossing of AKA was located on the left side in $86 \%$ of cases, between T8 and T10 in $73.33 \%$ of cases and L1-L2 in $26.67 \%$ of cases(5). The authors further suggested a classification, including the descending branch of AKA which was a continuation of ASA (type I) and ASA at the top of the arch (type II). The type I and its variant correlated to high neurological risk were present in $93.33 \%$ of cases, while the type II, correlated to medium or low neurological risk, was present in $6.67 \%$ of cases(5).

\section{Technical pitfalls}

A first aspect is related to preoperative neuroimaging, which might be more challenging for some specific conditions, including coexistent bony abnormalities, such as scoliosis, osteophytes, narrow intervertebral foramen or extensive arterial disease $(4,6)$.

A second important aspect is to change the surgical side if the AKA and the initially planned approach are on the same flank (please see below, our illustrative cases 1 and 2).

A third is to distinguish the AKA from the anterior radicuomedullary vein, the former being similar in shape and might closely follow the course of the AKA. A simple way to avoid confusion is to apply the so-called "continuity technique", while tracing the artery of Adamkiewicz from the aorta(7).

\section{Clinical syndrome}

Injury of the AKA results in anterior cord syndrome, frequently related to interrupted supply of the anterior spinal artery or the AKA (its major supplier), having as the major cause 
the atherosclerotic disease (or posttraumatic, multiple sclerosis, infectious). However, other possible cause is iatrogenic, occurring due to surgical approaches.

The anterior two-third of the spinal cord include the majority of anterior and lateral white matter funiculi, the central grey matter, the bilateral lateral and anterior horns, and the basis of posterior horns. Clinically, patients present with neurological deficit specific to the relevant structures: 1- corticospinal tract, bilateral loss of motor function (spastic paralysis below the lesion, flaccid at the level of the lesion); 2- spinothalamic tract, bilateral loss of pain and temperature one level below the lesion; 3- descending autonomic tracts, sexual dysfunction and urinary and fecal incontinence. The posterior columns are spared and so touch, vibration and proprioception remain intact. Motor recovery occurs in less than half of the patients(8).

\section{Illustrative cases with AKA on the same level and site as the treated pathology \\ Illustrative case 1 (figure 2)}

Forty-two years old male presented with left hip pain, with further intractable cruralgia on the left side, with continuous exacerbation during the past months. Clinical exam revealed L2 hypoesthesia. He didn't have any lack of force, nor cauda equina signs.

Lumbar MRI revealed a left intradural extramedullary, with intraforaminal expansion lesion, located at the L1-L2 level, with homogenous enhancement (Figure 2, A). Preoperative diagnosis was neurinoma. Spinal angiography showed the Adamkievicz's artery located at left L1 level.

Intracapsular decompression was proposed and performed.

Postoperative clinical exam was unremarkable, with disappearance of pain and a resumed physical exercise.

Six months follow-up MRI showed minimal residual tumor (2, B, with further proposal for "wait-and-scan" strategy.

\section{Illustrative case 2 (figure 3 )}

Forty years old patient presented with left intercostal intractable T6 neuralgia during the past 6 months.

MRI revealed a left T6-T7 intraforaminal lesion, with homogenous enhancement (Figure 3, A). Radiological diagnosis was neurinoma. Spinal angiography showed the Adamkievicz's artery located at left T6 level, same as the tumor. 
A radiosurgical treatment was proposed and performed by Cyberknife (Accuray) radiosurgery (Figure 3, B).

Following radiosurgery, the intercostal neuralgia initially exacerbated during several weeks, and further disappeared 6 months later. One year follow-up MRI revealed tumor stability.

\section{Our recommendations}

An opened question is whether to perform or not a preoperative SA on a regular manner and in all spinal tumor cases.

We usually perform such an investigation if we observe vascular abnormalities on preoperative MRI, or if the surgical approach involves any level between T4 and L2, especially (but not exclusively) for anterior approaches.

We also consider embolization in cases of extramedullary tumors, which are hypervascular. Some authors might argue that a hypervascular tumor is more likely to recruit feeding vessels from major radiculomedullary arteries supplying the spinal cord. Moreover, the thoracic spine is, for instance, the most frequent site of the metastatic disease, suggesting that the AKA may frequently be involved. Such connection would place the patient at risk for having a stroke during an embolization procedure. There is, however, no demonstration that hypervascular spinal metastatic tumors are associated with major arterial feeders to the spinal cord and some authors (9) suggested that using SA during preoperative embolization procedures, only $11.8 \%$ had an associated radiculomedullary arteries.

For spinal tumor where the AKA is exactly on the same level and side, an intracapsular decompression can be safely performed, with an immediate decrease of the symptomatic mass effect, while decreasing the risk of neurological injury. A second valuable alternative in such situations can also be radiosurgery, for small to medium size tumors. In our experience, this proved safe and effective both for tumor and pain control in the illustrative case 2 . 


\section{Compliance with Ethical Standards: yes}

Funding: Funding: Constantin Tuleasca gratefully acknowledges receipt of a 'Young Researcher in Clinical Research Grant' (Jeune Chercheur en Recherche Clinique) from the University of Lausanne (UNIL), Faculty of Biology and Medicine (FBM) and the Lausanne University Hospital (CHUV)

Conflict of interest: All authors certify that they have no affiliations, with or involvement in any organization or entity with any financial interest (such as honoraria; educational grants; participation in speakers' bureaus; membership, employment, consultancies, stock ownership, or other equity interest; and expert testimony or patent-licensing arrangements), or non-financial interest (such as personal or professional relationships, affiliations, knowledge or beliefs) in the subject matter or materials discussed in this manuscript.

Ethics approval: Ethical Committee Approval has been required, according to the French rules, at the Assistance Publique, Hopitaux de Paris, Paris, France for this retrospective review of cases. 


\section{References}

1. Lindeire S, Hauser JM. Anatomy, Back, Artery Of Adamkiewicz. StatPearls. Treasure Island (FL)2019.

2. Amato ACM, Parga Filho JR, Stolf NAG. Predictors of Adamkiewicz artery and anterior spinal artery detection through computerized tomographic angiography. SAGE open medicine. 2017;5:2050312117711599. PubMed PMID: 28616230. Pubmed Central PMCID: 5459350.

3. Grelat M, Madkouri R, Tremlet J, Thouant P, Beaurain J, Mourier KL. Aim and indications of spinal angiography for spine and spinal cord surgery: Based on a retrospective series of 70 cases. Neuro-Chirurgie. 2016 Feb;62(1):38-45. PubMed PMID: 26708079.

4. Charles YP, Barbe B, Beaujeux R, Boujan F, Steib JP. Relevance of the anatomical location of the Adamkiewicz artery in spine surgery. Surgical and radiologic anatomy : SRA. 2011 Jan;33(1):3-9. PubMed PMID: 20589376.

5. N'Da H A, Chenin L, Capel C, Havet E, Le Gars D, Peltier J. Microsurgical anatomy of the Adamkiewicz artery-anterior spinal artery junction. Surgical and radiologic anatomy : SRA. 2016 Jul;38(5):563-7. PubMed PMID: 26627692.

6. Yadav N, Pendharkar H, Kulkarni GB. Spinal Cord Infarction: Clinical and Radiological Features. Journal of stroke and cerebrovascular diseases : the official journal of National Stroke Association. 2018 Oct;27(10):2810-21. PubMed PMID: 30093205.

7. Murthy NS, Maus TP, Behrns CL. Intraforaminal location of the great anterior radiculomedullary artery (artery of Adamkiewicz): a retrospective review. Pain medicine. 2010 Dec;11(12):1756-64. PubMed PMID: 21134118.

8. Foo D, Rossier AB. Anterior spinal artery syndrome and its natural history. Paraplegia. 1983 Feb;21(1):1-10. PubMed PMID: 6835686.

9. Westbroek EM, Ahmed AK, Pennington Z, Goodwin ML, Xia Y, Boone C, et al. Hypervascular Metastatic Spine Tumor Angiographic Relationships with the Artery of Adamkiewicz and Other Radiculomedullary Arteries. World neurosurgery. 2019 Feb 28. PubMed PMID: 30825630. 


\section{Figures:}

Figure 1: indications for angiography, in our experience: vascular lesions (Figure 1, A), endocanalar juxtaforaminal lesions (Figure 1, B), foraminal lesions (Figure 1, C) or dumbell lesions (Figure 1, D)

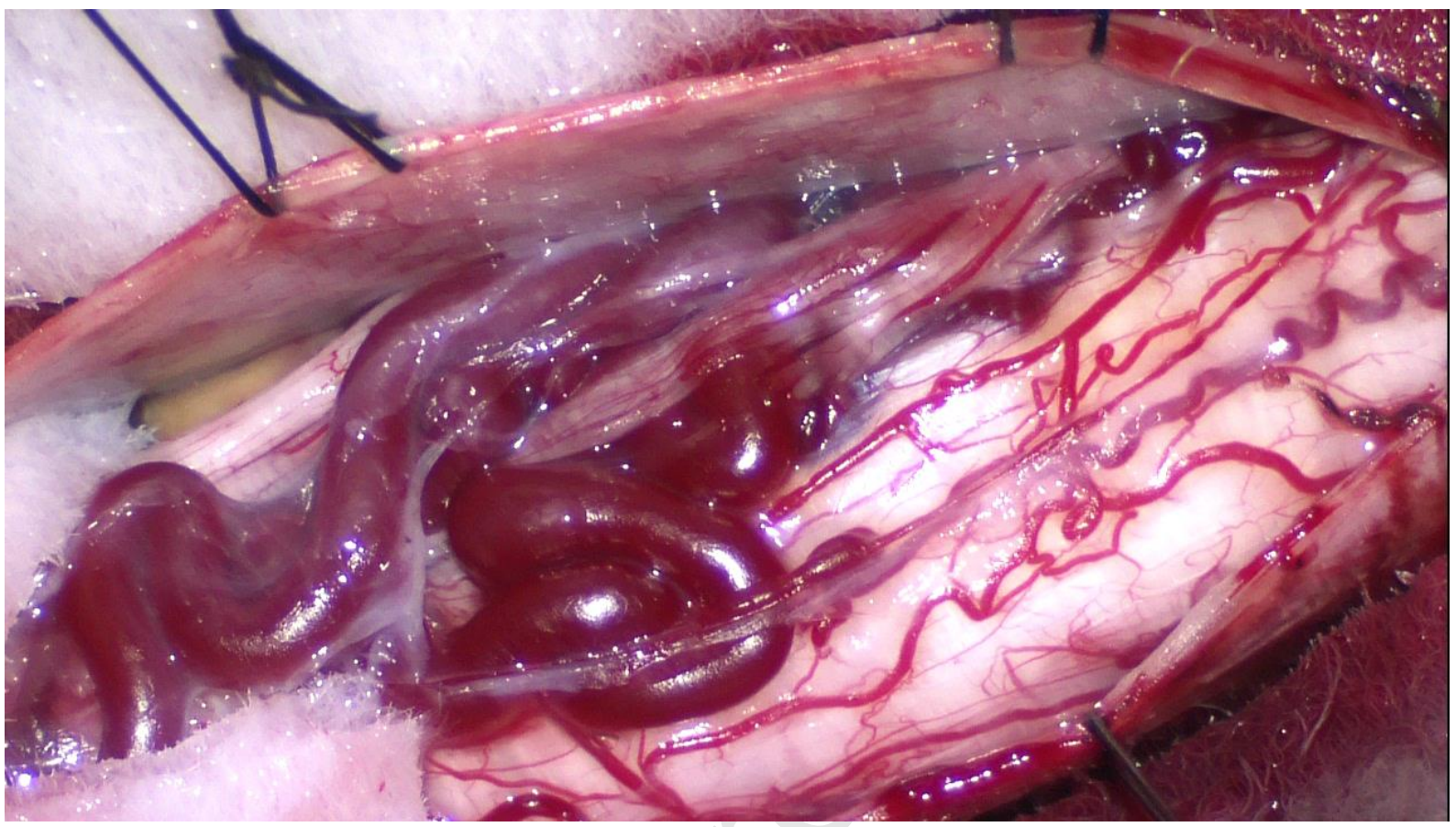


Figure 2: illustrative case 1- A: preoperative imaging with, from left to right, sagittal and axial T2 and sagittal and axial T1 Gadolinium injected MRI; spinal angiography (left) confirming the Adamkievicz artery on the left side and L1 level; B: 6 months postoperative MRI, with axial T2, sagittal and axial T1 Gadolinium injected MRI, showing minimal residual tumor

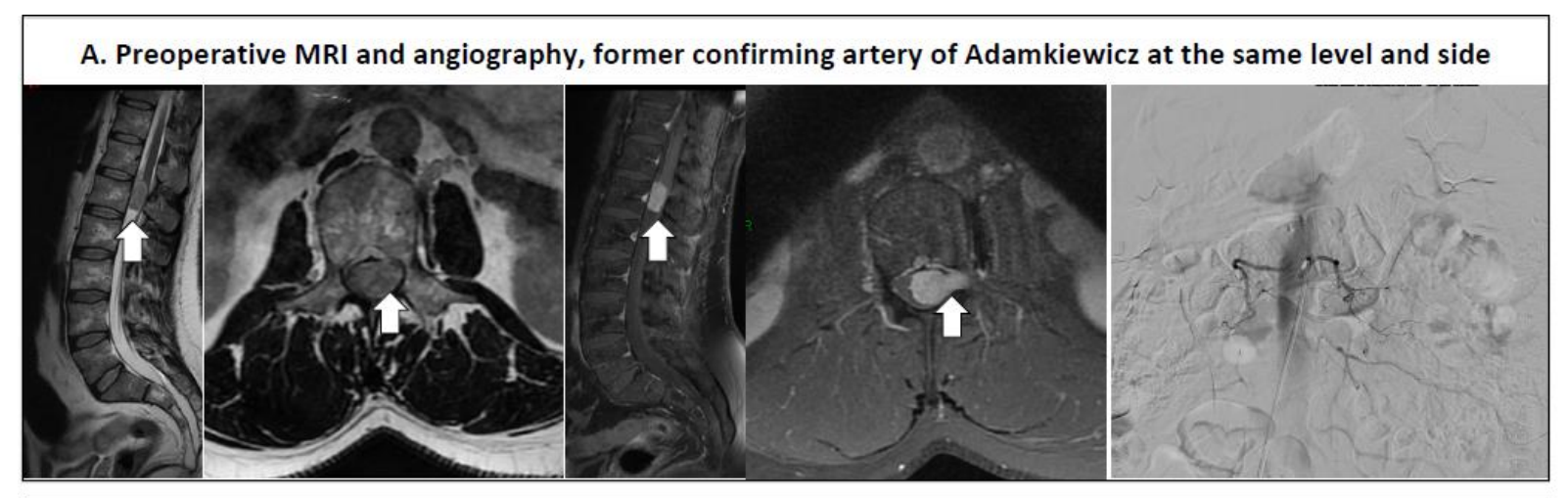

B. 6 months postoperative MRI after intracapsular decompression

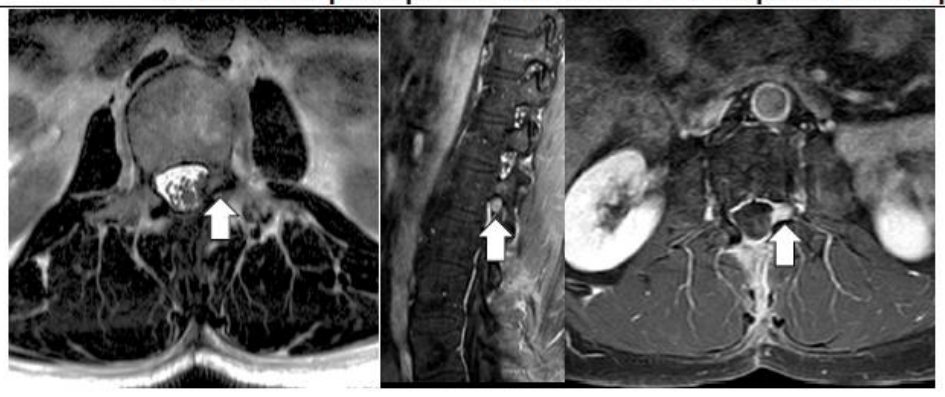


Figure 3: illustrative case 2- A: preoperative axial and sagittal MRI and preoperative spinal angiography; B: radiosurgical planning (courtesy Thomas Lacornerie, Center Oscar Lambret, Lille, France)
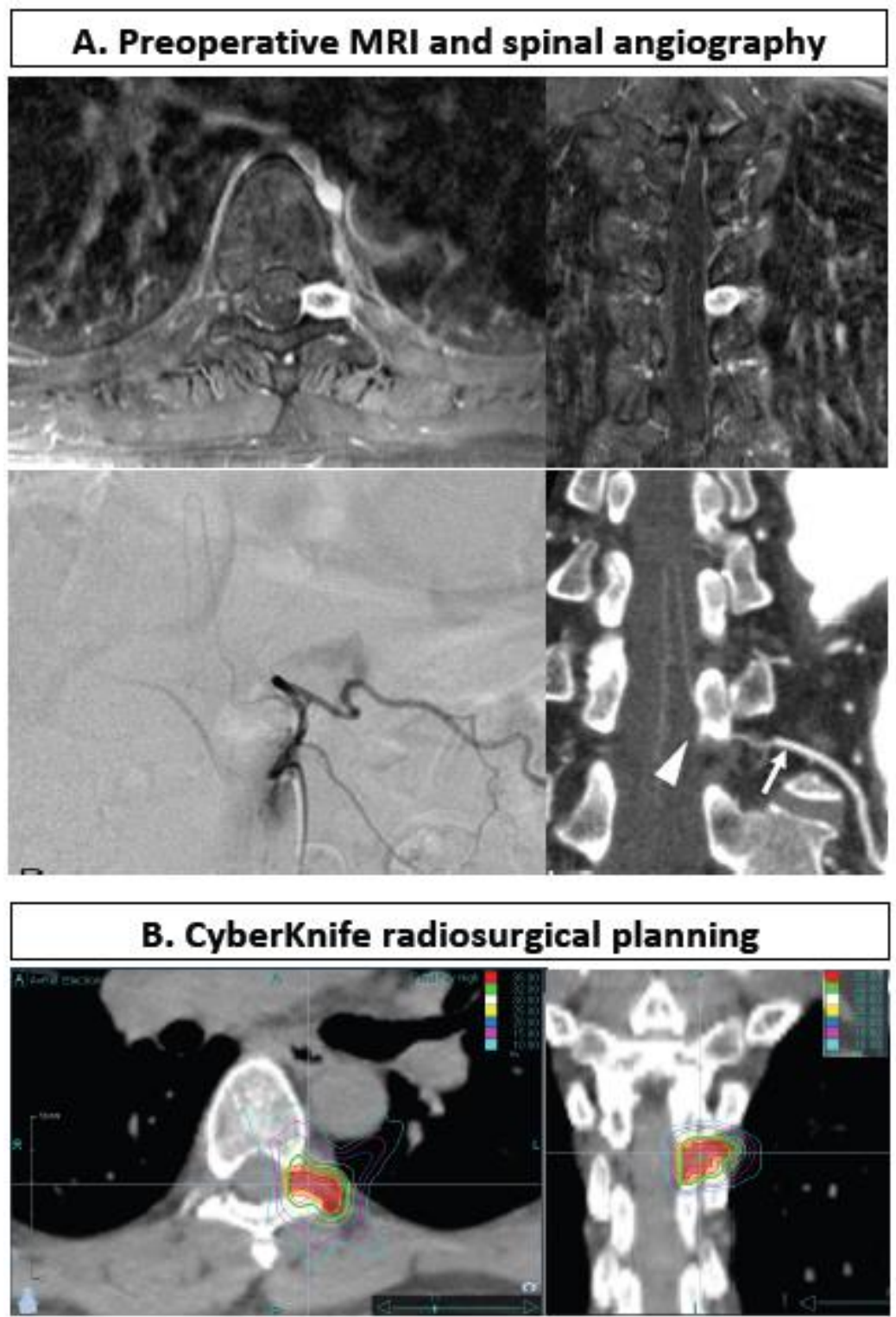